\title{
Morphology of Ni ultrathin films on Mo(110) and W(100) studied by LEED and STM
}

\author{
S. Murphy ${ }^{1}$, V. Usov, I.V. Shvets \\ Centre for Research on Adaptive Nanostructures and Nanodevices (CRANN), \\ School of Physics, Trinity College, Dublin 2, Ireland
}

\begin{abstract}
The morphology of ultrathin Ni films on $\mathrm{Mo}(110)$ and $\mathrm{W}(100)$ has been studied by low-energy electron diffraction and scanning tunneling microscopy. Ni films grow pseudomorphically on $\mathrm{Mo}(110)$ at $300 \mathrm{~K}$ for a coverage of $0.15 \mathrm{ML}$. A $(8 \times 1)$ structure is found at $0.4 \mathrm{ML}$, which develops into a $(7 \times 1)$ structure by $0.8 \mathrm{ML}$ as the film undergoes a structural change to fcc $\mathrm{Ni}(111)$ at $6 \mathrm{ML}$. The growth mode switches from layer-by-layer to Stranski-Krastanov between $4 \mathrm{ML}$ and $6 \mathrm{ML}$. Annealing at around $850 \mathrm{~K}$ results in alloying of submonolayer films with the substrate, while for higher coverages the $\mathrm{Ni}$ agglomerates into nanowedge islands. Ni films grow pseudomorphically on $\mathrm{W}(100)$ up to a coverage of around $2 \mathrm{ML}$ at $300 \mathrm{~K}$, above which there is a structural change from bcc to hcp Ni with the epitaxial relationship $(11 \overline{2} 0)_{N i} \|(100)_{W}$. This is accompanied by the formation of orthogonal domains of uniaxial strain-relieving dislocations from the third layer of the film. For coverages up to 1 ML the growth proceeds by formation of two-dimensional islands, but shifts to three-dimensional growth by $2 \mathrm{ML}$ with rectangular islands aligned along the $\langle 011\rangle$ substrate directions. Annealing at around $550 \mathrm{~K}$ results in agglomeration of $\mathrm{Ni}$ into larger islands and increasing film roughness.
\end{abstract}

Key words: scanning tunneling microscopy, molybdenum, tungsten, nickel, epitaxy PACS: 68.37.Ef, 61.14.Hg, 68.55.Jk

\section{Introduction}

The growth of ultrathin films of ferromagnetic transition metals on refractory metal surfaces has attracted much attention with respect to understanding

$\overline{1}$ Corresponding Author. Fax: + 3531896 3228; e-mail: shmurphy@tcd.ie 
the effects of epitaxial mismatch and film-substrate electron transfer on film morphology, magnetic properties and chemical reactivity. We have previously studied the growth, reactivity and magnetic properties of Fe ultrathin films on $\mathrm{Mo}(110)$ and compared them to the morphologically similar Fe/W(110) epitaxial system [1-5]. In the present study, we investigate the growth of $\mathrm{Ni}$ ultrathin films on $\mathrm{Mo}(110)$ by scanning tunneling microscopy (STM) and lowenergy electron diffraction (LEED) and compare the results with the better known $\mathrm{Ni} / \mathrm{W}(110)$ epitaxial system.

The Ni/W(110) system has been well-documented by both STM and LEED [6-8]. Ni grows in the Nishiyama-Wassermann epitaxial orientation on W(110), where the $\mathrm{Ni}(111)$ plane lies parallel to the $\mathrm{W}(110)$ surface and the $\mathrm{Ni}[1 \overline{1} 0]$ direction is aligned along the $\mathrm{W}[001]$ direction. The films initially grow pseudomorphically, giving rise to a $(1 \times 1)$ LEED pattern. However, the pseudomorphic layer is replaced by a $(8 \times 2)$ coincidence structure above a coverage of $0.2 \mathrm{ML}$. This transforms into a $(7 \times 2)$ coincidence structure above 0.4 ML. It should be noted that these $(8 \times 2)$ and $(7 \times 2)$ coincidence structures [6], are identified in later studies as $(8 \times 1)$ and $(7 \times 1)$ structures $[7,8]$ and will be referred to as such in the present study ${ }^{2}$. The formation of the coincidence structures reduces the large anisotropic strain and the fully relaxed $\mathrm{Ni}(111)$ layer is obtained for coverages above roughly 1.5 ML. The Ni films grow layer-by-layer at room temperature up to a coverage of $6 \mathrm{ML}$, whereupon there is a transition to three dimensional growth. The resulting islands have an hexagonal shape with island edges oriented along the $\langle 110\rangle$ directions [8]. Annealing at elevated temperatures results in Stranski-Krastanov growth with nanowedge islands supported on a closed monolayer, analagous to the case for $\mathrm{Fe} / \mathrm{W}(110)$ [9]. Recently, an experimental study combining simultaneous surface X-ray diffraction and stress measurements has demonstrated that a considerable rearrangement of the W(110) surface atoms takes place upon deposition of the $\mathrm{Ni}(111)$ overlayer [10]. Like the $\mathrm{Ni} / \mathrm{W}(110)$ system, $\mathrm{Ni} / \mathrm{Mo}(110)$ grows in the Nishiyama-Wassermann epitaxial orientation [11] and also undergoes a $(1 \times 1) \rightarrow(8 \times 1) \rightarrow(7 \times 1)$ series of structural transitions for submonolayer coverage at room temperature [12-14]. The growth proceeds in a layer-by-layer fashion at $115 \mathrm{~K}$ and $300 \mathrm{~K}$, with agglomeration into three-dimensional clusters and alloying occurring upon annealing above $600 \mathrm{~K}[12,15]$.

2 These LEED patterns can also be described with respect to the primitive surface net vectors of the substrate by the matrix representations $\left(\begin{array}{cc}1 & 1 \\ -4 & 4\end{array}\right)$ and $\left(\begin{array}{cc}4 & -3 \\ -3 & 4\end{array}\right)$, respectively. 
In addition, we have performed STM and LEED measurements on the Ni/W(100) epitaxial system. Previous studies of the Ni/W(100) by LEED, Auger electron spectroscopy (AES) and low-energy ion scattering (LEIS) have indicated that the first and second Ni layers are pseudomorphic and grow layer-by-layer at a substrate temperature of $100-300 \mathrm{~K}[16,17]$. Annealing at temperatures $\geq 700 \mathrm{~K}$ leads to intermixing of $\mathrm{Ni}$ and $\mathrm{W}$ at submonolayer coverages, though a pure $\mathrm{Ni}$ layer is stabilised at $1 \mathrm{ML}$ due to the lower surface energy of $\mathrm{Ni}$ [17]. Annealing the pseudomorphic bilayer results in intermixing in the first Ni layer, but negligible intermixing in the second. For higher coverages, there appears to be a switch to three-dimensional growth with the formation of islands of bulk Ni.

\section{Experimental}

The experiments were performed in a multichamber ultra-high vacuum (UHV) system with a base pressure in the low $10^{-10}$ Torr. The sample cleaning and film deposition were performed in a preparation chamber, equipped with a resistive heater for annealing samples to $900 \mathrm{~K}$, an electron-beam heater for annealing samples to temperatures reaching $2500 \mathrm{~K}$, an ISIS3000 ion gun (PSP Vacuum Technology) for ion etching samples and STM tips, variable leak valves for introducing $\mathrm{Ar}$ and $\mathrm{O}_{2}$, and an EFM 3 electron-beam evaporator (Omicron Nanotechnology Ltd.) for depositing the Ni films. The sample characterisation was performed in adjoining analysis chambers, equipped with a SPECTALEED (Omicron) four-grid front-view LEED optics, a cylindrical mirror analyser for AES (Physical Electronics) and a home-built roomtemperature STM based on a design by Pan et al. [18], which is controlled by a SCALA (Omicron) control unit. The LEED patterns were recorded with a Dragonfly digital camera (Point Grey Research) mounted in an enclosure in front of the phosphor screen.

The Mo substrate used was a $4 \mathrm{~N}$ purity single crystal with a miscut of $0.65^{\circ}$ from the (110) crystal plane. The $\mathrm{W}$ substrate was prepared from a $5 \mathrm{~N} \mathrm{pu-}$ rity single crystal, which was aligned to within $\pm 0.5^{\circ}$ of the (100) crystal plane. The samples were cleaned in-vacuum by annealing in $5 \times 10^{-7}$ Torr $\mathrm{O}_{2}$ at $1400 \pm 100 \mathrm{~K}$ for $30-60 \mathrm{~min}$, followed by flash-annealing several times to $2400 \pm 100 \mathrm{~K}$ for $10-15 \mathrm{~s}$ under UHV conditions. This was repeated until oxygen was the only remaining impurity, which was then removed by repeated flash-annealing in UHV. The sample temperature was monitored during the cleaning process using an infra-red pyrometer (Ircon Ultimax UX-20P) with an emissivity value of 0.35 . Ni films were deposited on the clean surfaces at rates in the range of $0.02-0.4 \mathrm{ML} / \mathrm{min}$ by electron-beam evaporation of a $99.99 \%$ purity Ni rod, which was extensively outgassed prior to use. A quartz crystal balance was used to monitor the deposition rate and the film thickness. 
The chamber pressure did not exceed $6 \times 10^{-10}$ Torr during evaporation. Film coverages were determined from the deposited film thickness and are given in pseudomorphic monolayers $(\mathrm{ML})$, where $1 \mathrm{ML}=1.43 \times 10^{19}$ atoms $/ \mathrm{m}^{2}$ for $\mathrm{Mo}(110)$ and $9.98 \times 10^{18}$ atoms $/ \mathrm{m}^{2}$ for $\mathrm{W}(100)$. The films were typically deposited at room temperature and then post-annealed in the resistive heater, which was equipped with a K-type thermocouple to measure the sample temperature. The STM images were obtained at room temperature in constant current mode using $\mathrm{W}$ tips, with a tunnel current of $0.1 \mathrm{nA}$ and a sample bias of $30 \mathrm{mV}$. The STM images were processed using WSxM [19].

\section{Results and discussion}

Figure 1 shows STM and LEED images of the clean Mo(110) and W(100) surfaces. The Mo(110) surface was characterised by atomic terraces that were on average 10-20 nm wide, which were separated by monatomic steps aligned along the $[1 \overline{1} \overline{1}]$ direction. The $\mathrm{W}(100)$ surface comprised 5-70 $\mathrm{nm}$ wide terraces separated by [011]-oriented monatomic steps ${ }^{3}$. In both cases, the clean surface displayed a $(1 \times 1)$ LEED pattern consistent with the unreconstructed bulk termination.

\section{$3.1 \mathrm{Ni} / \mathrm{Mo}(110)$}

The growth of $\mathrm{Ni}$ on $\mathrm{Mo}(110)$ at room temperature is summarised in Fig. 2, which shows a series of STM images of films in the 0.15 ML to 11.6 ML coverage range. Submonolayer growth is characterised by the formation of twodimensional islands on the substrate terraces and along the lower step edges [Fig. 2(a)]. These islands coalesce with increasing coverage [Fig. 2(b)] to form a continuous two-dimensional layer [Fig. 2(c)]. The growth of the second $\mathrm{Ni}$ layer proceeds in the same manner and is almost completely closed before third layer islands begin to nucleate [Fig. 2(d)]. The film roughness increases at higher coverages, as can be seen in Figs. 2(e) and (f), which show Ni films with 3.9 ML and 6.2 ML coverage, respectively. In the latter case, several layers of the film can be observed at the same time on the surface, and the growth mode can therefore be classified as three-dimensional. At even higher coverages [Figs. 2(g) and (h)] the film morphology is typified by "cascades" of $\mathrm{Ni}$ islands, which are formed by the overlap of islands on successive terraces and are approximately oriented along the miscut direction of the substrate.

3 The height of the steps on the Mo(110) surface was measured by STM to be $1.9 \pm 0.3 \AA$, while those on the $\mathrm{W}(100)$ surface were measured to be $1.6 \pm 0.3 \AA$. 


\begin{tabular}{|c|c|c|c|c|c|c|}
\hline Ni monolayers & 0.2 & 0.4 & 0.8 & $1.5 \quad 3.9$ & 6.2 & $9.2 \quad 11.6$ \\
\hline LEED pattern & $(1 \times 1)$ & $(8 \times 1)$ & & $(7 \times 1)$ & & $\mathrm{Ni}(111)$ \\
\hline strain along $\mathrm{Mo}[001]$ & $26.3 \%$ & $1.0 \%$ & & $-1.8 \%$ & & $0 \%$ \\
\hline strain along $\mathrm{Mo}[1 \overline{1} 0]$ & $3.1 \%$ & $3.1 \%$ & & $3.1 \%$ & & $0 \%$ \\
\hline
\end{tabular}

Table 1

Summary of the observed LEED patterns and estimated strain for different coverages of $\mathrm{Ni}$ deposited onto $\mathrm{Mo}(110)$ at $300 \mathrm{~K}$.

Figure 3 shows the LEED patterns for submonolayer Ni films deposited on $\mathrm{Mo}(110)$ at $300 \mathrm{~K}$. At $0.15 \mathrm{ML}$, the $\mathrm{Ni}$ film grows pseudomorphically on the $\mathrm{Mo}(110)$ surface as illustrated by the $(1 \times 1)$ LEED pattern shown in Figs. 3(a) and (b) for different beam energies. As mentioned earlier, Ni/Mo(110) grows in the Nishiyama-Wassermann orientation [11], with the epitaxial relationship $(111)_{N i} \|(110)_{M o}$, where $[1 \overline{1} 0]_{N i} / /[001]_{M o}$ and $[11 \overline{2}]_{N i} \|[1 \overline{1} 0]_{M o}$. The $\mathrm{Ni}(111)$ layer is distorted in this configuration with $26.3 \%$ tensile strain along $[001]_{M o}$ and $3.1 \%$ tensile strain along $[1 \overline{1} 0]_{M o}$. With such a massive strain it is not surprising that the film relaxes in the submonolayer coverage regime, giving rise to the previously reported $(1 \times 1) \rightarrow(8 \times 1) \rightarrow(7 \times 1)$ series of structural transitions [12-14]. At a coverage of $0.4 \mathrm{ML}$, the film develops a $(8 \times 1)$ LEED pattern with additional spots along the $[00 \overline{1}]_{M o}$ direction $[$ Figs. $3(\mathrm{~d})$ and (e)]. A schematic illustration of this pattern is shown in Fig. 3(f). This corresponds to the formation of a periodic superlattice, shown schematically in Fig. 4(a), which is aligned along $[1 \overline{1} 0]_{M o}$ and where there are five $\mathrm{Ni}-\mathrm{Ni}$ bond lengths for every four Mo-Mo bond lengths along $[001]_{M o}$. The tensile distortion of the $\mathrm{Ni}(111)$ layer in this case is $1.0 \%$ along $[001]_{M o}$ and remains $3.1 \%$ along $[1 \overline{1} 0]_{M o}$. At 0.8 ML the $(7 \times 1)$ LEED pattern shown in Figs. 3(g-i) was observed. A schematic model of the corresponding real space superlattice is shown in Fig. 4(b). In this case, the $\mathrm{Ni}(111)$ layer is distorted along [001 $]_{M o}$ so that there are nine $\mathrm{Ni}-\mathrm{Ni}$ bond lengths for every seven Mo-Mo bond lengths. This results in a slight compressive strain of $1.8 \%$ along [001 $]_{M o}$, while the film strain remains the same along $[1 \overline{1} 0]_{M o}$. It should be noted that the $(8 \times 1)$ or $(7 \times 1)$ structures were not resolved with STM. Figure 5 shows the LEED patterns obtained from multilayer $\mathrm{Ni}$ films deposited on $\mathrm{Mo}(110)$ at $300 \mathrm{~K}$. It was observed that films in the coverage range between $0.8 \mathrm{ML}$ and $6.2 \mathrm{ML}$ display a $(7 \times 1)$ LEED pattern $[$ c.f. Figs. $3($ g-i) for a 0.8 ML film and Figs. 5(a-e) for 1.5 ML and 6.2 ML films, respectively]. However, in the case of the 6.2 ML film the $(7 \times 1)$ pattern was only observed at higher energies, while at lower energies only reflections consistent with a relaxed $\mathrm{Ni}(111)$ layer were observed. This indicates that the uppermost layers of this film were fully relaxed $\mathrm{Ni}(111)$. For higher coverages, only a $(1 \times 1)$ hexagonal LEED pattern corresponding to relaxed $\mathrm{Ni}(111)$, like that shown in Fig. 5(f-g), was observed. A summary of the structural evolution of the films deposited at $300 \mathrm{~K}$ is presented in Table 1. 
Figure 6 shows a series of STM images and LEED patterns of 4.6 ML films deposited at room temperature and post-annealed for $1 \mathrm{~h}$ in UHV at $575 \mathrm{~K}$ and $750 \mathrm{~K}$. The film deposited at $300 \mathrm{~K}$ [Fig. 6 (a)] follows the general trend displayed by the films in Fig. 2. The corresponding LEED pattern [Fig. 6(b)] shows faint satellite spots consistent with the $(7 \times 1)$ structure. Annealing at $575 \mathrm{~K}$ does not have a substantial effect on the morphology, other than some island coalescence and general roughening of the film [Fig. 6 (c)]. This demonstrates that the adatom mobility is far lower in the $\mathrm{Ni} / \mathrm{Mo}(110)$ system compared to the $\mathrm{Fe} / \mathrm{Mo}(110)$ system, as in the latter case annealing at $575 \mathrm{~K}$ results in agglomeration of Fe into nanowedge islands [2]. No satellite spots were observed for the film annealed at $575 \mathrm{~K}$ [Fig. 6 (d)], which seems to be due to the increased roughness of the film resulting in a more diffuse LEED pattern. Annealing at $750 \mathrm{~K}$ is needed to produce a substantial change in film morphology. Figure 6(e) shows how the Ni agglomerates to form smooth step fronts that are $3.1 \AA$ high. The corresponding LEED pattern of the surface [Fig. 6 (f)] displays sharp $(7 \times 1)$ spots. A larger area STM image [Fig. 6 (g)] reveals that the $\mathrm{Ni}$ accumulates patches on the surface, leaving some areas of the substrate exposed.

Figure 7 shows STM and LEED images of 0.15-2.3 ML films deposited at $300 \mathrm{~K}$ and post-annealed for $1 \mathrm{~h}$ at $825-855 \mathrm{~K}$. Annealing a $0.15 \mathrm{ML}$ film results in the formation of step edges that are faceted predominantly along the [001] and [11ㅣ] directions, c.f Fig. 7(a). Furthermore, the Ni film forms finger-like protrusions along the [001] direction. The annealed film also produces a $c(2 \times 2)$ LEED pattern [Fig. $7(b)]$, which is attributed to the formation of a surface alloy as it has been established that alloying occurs in the $\mathrm{Ni} / \mathrm{Mo}(110)$ epitaxial system for temperatures above $700 \mathrm{~K}$ [12]. Annealing a $0.8 \mathrm{ML}$ film at $855 \mathrm{~K}$ results in the coalescence of $\mathrm{Ni}$ on each substrate terrace into large two-dimensional islands [Fig. 7(c)], while annealing a 2.3 ML film at $825 \mathrm{~K}$ produces the nanowedge islands [Fig. $7(\mathrm{e})$ ], previously observed in the $\mathrm{Ni} / \mathrm{W}(110), \mathrm{Fe} / \mathrm{Mo}(110)$ and $\mathrm{Cr} / \mathrm{Mo}(110)$ systems $[2,8,20]$, which range in local thickness from 2-3 ML up to around 15 ML.

The STM data presented here agree well with the results of previous measurements of the $\mathrm{Ni} / \mathrm{Mo}(110)$ system using other techniques $[12,13,15]$. The STM results confirm earlier LEED, AES and workfunction measurements performed by Tikhov and Bauer [12] that the first few monolayers of $\mathrm{Ni}$ grow on $\mathrm{Mo}(110)$ in near layer-by-layer fashion, with increasing roughness apparent from the second monolayer onwards. The STM data also show that the morphology of multilayer Ni films does not change much when heated below $600 \mathrm{~K}$, but that the films agglomerate when heated to $750 \mathrm{~K}$, in agreement with AES measurements by He et al. $[13,15]$. However, we note that the films only agglomerate into three-dimensional islands with well-defined geometry, i.e. nanowedge islands, at temperatures in excess of $800 \mathrm{~K}$. The STM and LEED results presented in Fig. 7(a) and (b) also indicate that submonolayer Ni undergoes in- 
terfacial alloying at temperatures above $800 \mathrm{~K}$, which is in further agreement with the AES measurements made by He et al. [13,15]. As mentioned earlier, the LEED measurements reproduce the $(1 \times 1) \rightarrow(8 \times 1) \rightarrow(7 \times 1)$ series of structural transitions observed in the earlier studies $[12,13]$. However, we have found that the $(8 \times 1)$ and $(7 \times 1)$ patterns appear at much lower coverages than previously reported. For example, we have found that the $(8 \times 1) \rightarrow(7 \times 1)$ structural transition occurs in the coverage range between $0.4 \mathrm{ML}$ and $0.8 \mathrm{ML}$, whereas Tikhov and Bauer [12] located this in the 0.74 ML to 1.29 ML range, while He et al. [13] observed it in the $0.95 \mathrm{ML}$ to $1.08 \mathrm{ML}$ range. However, we have also found that the $(7 \times 1)$ LEED pattern persists up to $6 \mathrm{ML}$, which agrees with what was previously reported by He et al. [13].

The growth behaviour of the $\mathrm{Ni} / \mathrm{Mo}(110)$ system is similar to that of the $\mathrm{Ni} / \mathrm{W}(110)$ system [6-8]. In both cases, the initial growth proceeds in a layerby-layer fashion for the first 2-3 ML after which the film roughness increases with coverage, while three-dimensional growth is established at a coverage of around 6 ML. Both systems adopt the Nishiyama-Wasserman epitaxial orientation, and display a $(1 \times 1) \rightarrow(8 \times 1) \rightarrow(7 \times 1)$ series of LEED patterns in the submonolayer regime. The $(8 \times 1)$ and $(7 \times 1)$ LEED patterns are observed at lower coverages in the $\mathrm{Ni} / \mathrm{W}(110)$ system (0.2 ML and $0.4 \mathrm{ML}$, respectively) compared to the $\mathrm{Ni} / \mathrm{Mo}(110)$ due to the larger mismatch between the film and substrate. In both systems, annealing multilayer films to temperatures of around $800 \mathrm{~K}$ results in agglomeration into nanowedge islands [8]. Some differences are observed in the morphology of $\mathrm{Ni} / \mathrm{Mo}(110)$ grown here at $300 \mathrm{~K}$, compared to the Ni/W(110) films studied by Schmidthals et al.[8]. For example, in the latter study the formation of triangular islands with edges aligned along the $\mathrm{Ni}\langle 110\rangle$ directions was observed at coverages of around $10 \mathrm{ML}$, which was independent of deposition rate. By comparison, we did not observe triangular islands at coverages of $9 \mathrm{ML}$ or higher [c.f Figs. 2(g) and (h)] but rather "cascades" of islands. Moreover, we observe a much higher level of step decoration in the first and second layers compared to the $\mathrm{Ni} / \mathrm{W}(110)$ study. These differences can be attributed to the much higher step density on the Mo(110) surface used in the present study, compared to that observed on the $\mathrm{W}(110)$ surface in the $\mathrm{Ni} / \mathrm{W}(110)$ study, where the average terrace width was of the order of $65 \mathrm{~nm}$ [8]. The enhanced step decoration observed for the $\mathrm{Ni} / \mathrm{Mo}(110)$ films in the present case arised because the substrate terraces were sufficiently narrow that when the $\mathrm{Ni}$ adatoms are deposited they can diffuse to a step edge where they become incorporated. A small fraction of the adatoms nucleated on the terraces to form two-dimensional islands, which captured more and more adatoms as their size increased. Because of the narrow terrace width these islands quickly became larger than the terraces on which they were supported, and they overlapped with islands on adjacent terraces [c.f. Fig. 2(f)]. This formed the template for the subsequent growth of the "cascades" that were observed at higher coverages. In the case of the $\mathrm{Ni} / \mathrm{W}(110)$ study, the terraces were sufficiently large that the larger fraction of 
the deposited $\mathrm{Ni}$ adatoms formed or attached to two-dimensional islands before reaching a step. As a result, there was minimal step decoration and much larger two-dimensional islands formed on the substrate terraces. Subsequently deposited adatoms would have been able to diffuse over much longer paths on these islands without meeting an island edge compared to the $\mathrm{Ni} / \mathrm{Mo}(110)$ case. As a result, anisotropic diffusion kinetics related to the symmetry of the $\mathrm{Ni}(111)$ surface may have played a larger role in the $\mathrm{Ni} / \mathrm{W}(110)$ system, resulting in the observed triangular islands. It would be interesting in future to study the morphology of $\mathrm{Ni}$ films deposited under the same conditions on a $\mathrm{Mo}(110)$ substrate with a much lower miscut, to make a direct comparison with the Ni/W(110) study by Schmidthals et al. [8].

\section{2 $\mathrm{Ni} / \mathrm{W}(100)$}

The growth of Ni on W(100) at $300 \mathrm{~K}$, is summarised in Fig. 8. Submonolayer coverage films grow as monatomically high two-dimensional islands and display a sharp $(1 \times 1)$ LEED pattern consistent with pseudomorphic growth of bcc Ni. Pseudomorphic growth was observed by LEED up to nearly $2 \mathrm{ML}$ in agreement with earlier studies of the $\mathrm{Ni} / \mathrm{W}(001)$ system $[16,17]$. Figure 8(b) shows a STM image of a $1.8 \mathrm{ML}$ film deposited at $300 \mathrm{~K}$. The film forms three-dimensional rectangular islands that are oriented along the $\langle 011\rangle$ substrate directions. While the $1.8 \mathrm{ML}$ film shown in Fig. 8(b) displayed a $(1 \times 1)$ LEED pattern, in STM images of the surface we were able to resolve ripples with $11 \pm 1 \AA$ periodicity on the surface of some of the Ni islands. The ripples, shown in Fig. 8(c), are aligned along the [011] direction and only appear on the surface of islands that are locally $3 \mathrm{ML}$ or more thick. For higher film coverages the ripples become widespread across the surface, giving rise to a LEED pattern like that shown in Fig. 8(d), which was obtained on a $2.7 \mathrm{ML}$ film deposited at $300 \mathrm{~K}$. The pattern displays satellite spots around the $\langle 011\rangle$ reflections from the substrate, as well as half-order spots (with some additional fine-structure) arranged in a $\mathrm{c}(2 \times 2)$ pattern. A schematic model of the pattern is presented in Fig. $8(\mathrm{e})$. The appearance of the $\mathrm{c}(2 \times 2)$ pattern is consistent with a structural change in the film from bcc to hcp $\mathrm{Ni}$, with the epitaxial relation $(11 \overline{2} 0)_{N i} \|(100)_{W}$ [22]. In this configuration, the [0001] and [1100] directions of the film are aligned parallel to the $\langle 011\rangle$ directions of the substrate. Because the (1120) plane of hcp Ni is not perfectly square, this means that the epitaxial strain differs along the [0001] and [1ํㅣㄹ] directions in the film. The ripple structure observed on the surface of the $\mathrm{Ni}$ islands is the result of uniaxial strain relief along the [0001] direction in the film by the formation of [1100]-oriented dislocations [21]. As the film coverage is increased further the $\mathrm{Ni}$ islands become more elongated along the $\langle 01 \overline{1}\rangle$ substrate directions, c.f. Fig. 8(f). The film roughness increases so that the LEED pattern of the surface becomes diffuse. Finally, post-annealing the Ni/W(100) films 
at 550-575 $\mathrm{K}$ was found to result in coalescence of the $\mathrm{Ni}$ islands, producing rougher films that displayed diffuse LEED patterns.

The Ni/W(100) films presented here display many morphological similarities to the previously studied $\mathrm{Co} / \mathrm{W}(001)$ epitaxial system [21]. We have found that the $\mathrm{Ni} / \mathrm{W}(100)$ films grow pseudomorphically at $300 \mathrm{~K}$ up to about $2 \mathrm{ML}$, above which the film structure switches from bcc to hcp with the epitaxial relation $(11 \overline{2} 0)_{N i} \|(100)_{W}$, resulting in a $\mathrm{c}(2 \times 2)$ LEED pattern. Similarly, $\mathrm{Co} / \mathrm{W}(001)$ films grow pseudomorphically up to $2.7 \mathrm{ML}$ at $300 \mathrm{~K}$, above which they adopt a hcp structure with the $(11 \overline{2} 0)_{C o} \|(100)_{W}$ epitaxial relation, also resulting in a $c(2 \times 2)$ LEED pattern [21]. In both systems, the multilayer growth is characterised by the formation of three-dimensional islands elongated along the $\langle 110\rangle$ directions. In both cases, a ripple structure aligned along the $\langle 110\rangle$ directions was observed on the surface of the islands with STM, giving rise to splitting of the half-order spots in the $\mathrm{c}(2 \times 2)$ LEED pattern.

\section{Conclusions}

The $\mathrm{Ni} / \mathrm{Mo}(110)$ and $\mathrm{Ni} / \mathrm{W}(100)$ epitaxial systems display qualitatively similar growth behaviour to the $\mathrm{Ni} / \mathrm{W}(110)$ and $\mathrm{Co} / \mathrm{W}(001)$ systems, respectively. Ni films undergo a series of structural changes during submonolayer growth on $\mathrm{Mo}(110)$ at $300 \mathrm{~K}$ from pseudomorphic at a coverage of $0.15 \mathrm{ML}$, to a $(8 \times 1)$ structure at $0.4 \mathrm{ML}$, to a $(7 \times 1)$ structure at $0.8 \mathrm{ML}$. This last structure persists up to a coverage of $4-6 \mathrm{ML}$, where the film adopts a $(111)_{N i} \|(110)_{M o}$ epitaxial relation with the substrate. This final transition is accompanied by a transition from layer-by-layer to Stranski-Krastanov growth. Annealing the $\mathrm{Ni} / \mathrm{Mo}(110)$ films at around $850 \mathrm{~K}$ results in intermixing in the case of submonolayer films and the formation of $\mathrm{Ni}(111)$ nanowedge islands at higher coverages. Ni films grow pseudomorphically on W(100) up to a coverage of around $2 \mathrm{ML}$ at $300 \mathrm{~K}$. As soon as the local island thickness exceeds three layers there is a structural transition from bcc to hcp Ni with the epitaxial relation $(11 \overline{2} 0)_{N i} \|(100)_{W}$. At the same time uniaxial dislocations are formed along the $[1 \overline{1} 00]$ direction in the film.

\section{Acknowledgments}

This work was supported by Science Foundation Ireland (00/PI.1/C042) and the European project ASPRINT (NMP-CT-2003-001601). 


\section{References}

[1] J. Osing, S. Murphy, I.V. Shvets, Surf. Sci. 454-456 (2000) 280.

[2] S. Murphy, D. Mac Mathúna, G. Mariotto, I.V. Shvets, Phys. Rev. B 66 (2002) 195417.

[3] S. Murphy, G. Mariotto, N. Berdunov, I.V. Shvets, Phys. Rev. B 68 (2003) 165419.

[4] V. Usov, S. Murphy, I.V. Shvets, J. Appl. Phys. 95 (2004) 7312.

[5] V. Usov, S. Murphy, I.V. Shvets, J. Magn. Magn. Mater. 283 (2004) 357.

[6] J. Kołaczkiewicz, E. Bauer, Surf. Sci. 144 (1984) 495.

[7] C. Schmidthals, A. Enders, D. Sander, J. Kirschner, Surf. Sci. $402-404$ (1998) 636 .

[8] C. Schmidthals, D. Sander, A. Enders, J. Kirschner, Surf. Sci. 417 (1998) 361.

[9] H. Bethge, D. Heuer, Ch. Jensen, K. Reshöft, U. Köhler, Surf. Sci. 331-333 (1995) 878.

[10] H.L. Meyerheim, D. Sander, R. Popescu, J. Kirschner, O. Robach, S. Ferrer, P. Steadman, Phys. Rev. B 67 (2003) 155422.

[11] F. Martin, J. Pacaud, G. Abadias, C. Jaouen, P. Guérin, Appl. Surf. Sci. 188 (2002) 90 .

[12] M. Tikhov, E. Bauer, Surf. Sci. 232 (1990) 73.

[13] J.-W. He, W.K. Kuhn, D.W. Goodman, Surf. Sci. 292 (1993) 248.

[14] H. Tsunematsu, M. Aita, A. Tanaka, S. Suzuki, S. Sato, Y. Gotoh, J. Electron Spectrosc. Related Phenomena 101-103 (1999) 281.

[15] J.-W. He, W.-L. Shea, X. Jiang, D.W. Goodman, J. Vac. Technol. A 8 (1990) 2435.

[16] P.J. Berlowitz, D.W. Goodman, Surf. Sci. 187 (1987) 463.

[17] S.H. Overbury, P.F. Lyman, D.R. Mullins, N.D. Shinn, Surf. Sci. 339 (1995) 68.

[18] S.H. Pan, E.W. Hudson, J.C. Davis, Rev. Sci. Instrum. 70 (1999) 1459.

[19] I. Horcas, R. Fernandez, J.M. Gomez-Rodriguez, J. Colchero, J. Gomez-Herrero, A.M. Baro, Rev. Sci. Instrum. 78 (2007) 013705.

[20] A. Cazacu, S. Murphy, I.V. Shvets, Phys. Rev. B 73 (2006) 045413.

[21] W. Wulfhekel, T. Gutjahr-Löser, F. Zavaliche, D. Sander, J. Kirschner, Phys. Rev. B 64 (2001) 144422.

[22] H. Wormeester, E. Hüger, E. Bauer, Phys. Rev. Lett. 77 (1996) 1540. 

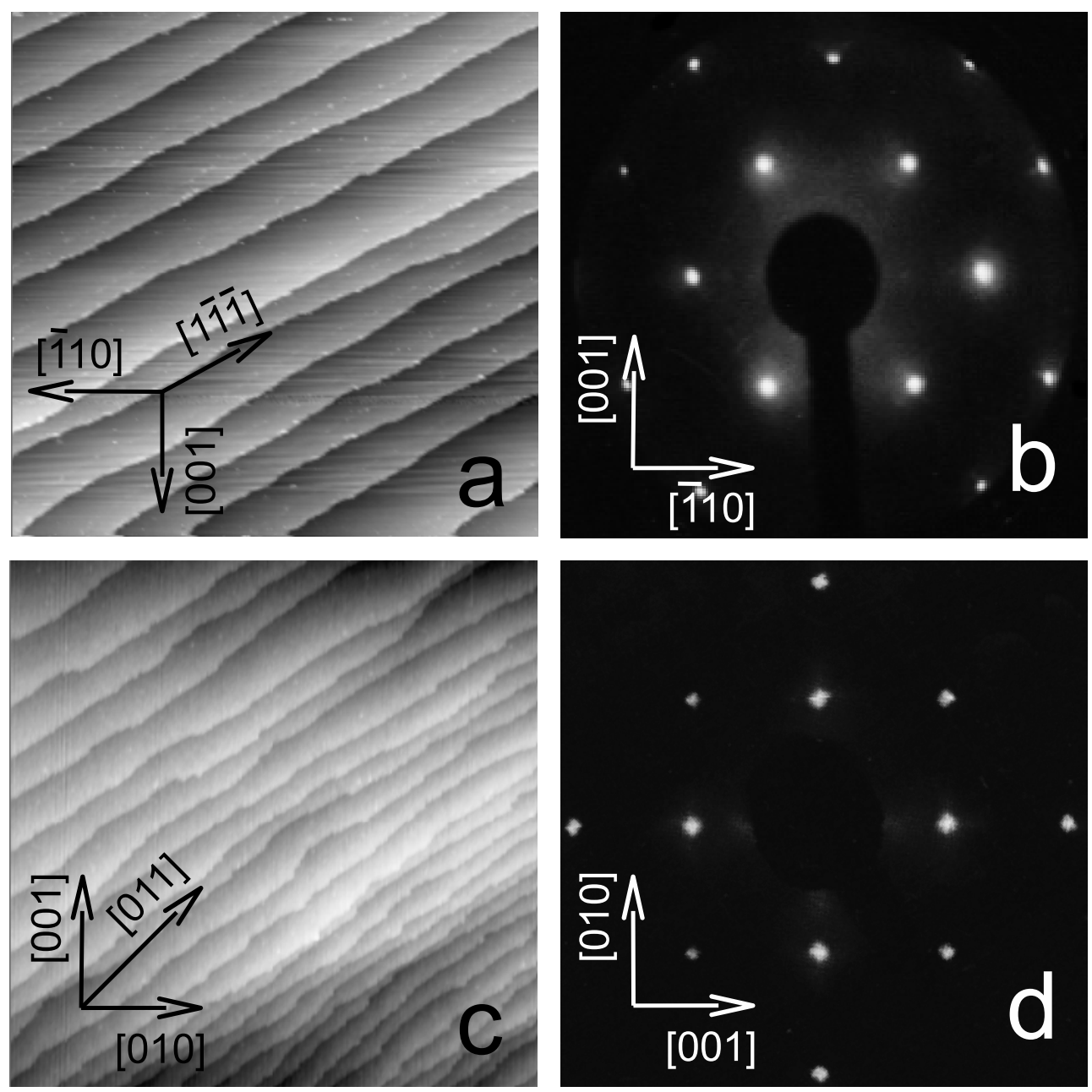

Fig. 1. (a) $200 \mathrm{~nm} \times 200 \mathrm{~nm}$ STM image of the uncovered Mo(110) surface, with the main crystallographic directions marked. (b) LEED pattern of the Mo(110) surface taken at $177 \mathrm{eV}$. (c) $200 \mathrm{~nm} \times 200 \mathrm{~nm}$ STM image of the uncovered W(001) surface, with the main crystallographic directions marked. (d) LEED pattern of the W(100) surface taken at $159 \mathrm{eV}$. 

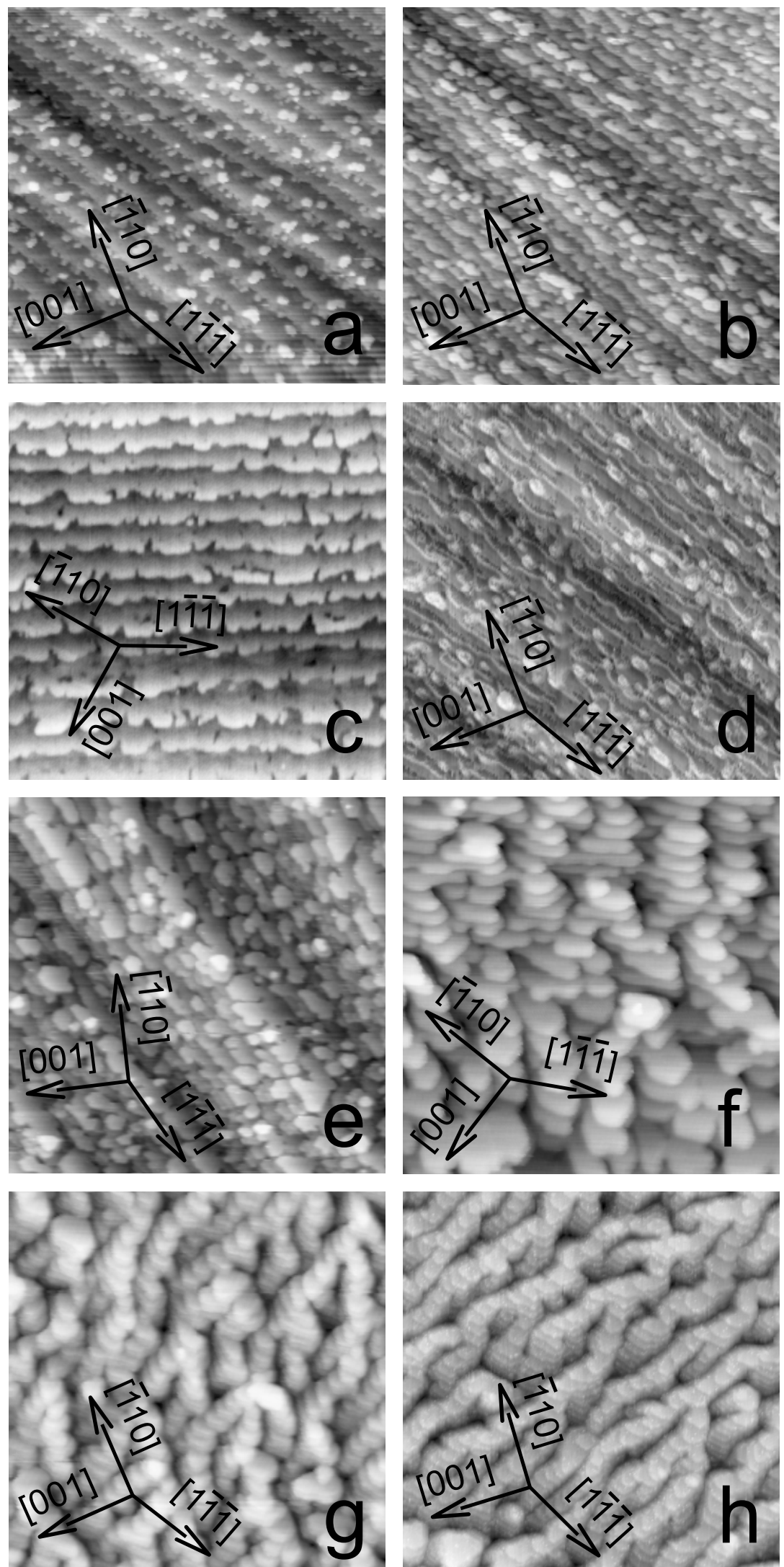

Fig. 2. $200 \mathrm{~nm} \times 200 \mathrm{~nm} \mathrm{STM}$ images of $\mathrm{Ni}$ films grown on $\mathrm{Mo}(110)$ at room temperature, with the main crystallographic axes of the substrate indicated. (a) $0.15 \mathrm{ML}$, (b) $0.4 \mathrm{ML}$, (c) $0.8 \mathrm{ML}$, (d) $1.5 \mathrm{ML}$, (e) $3.9 \mathrm{ML}$, (f) $6.2 \mathrm{ML}$, (g) $9.2 \mathrm{ML}$ and (h) 11.6 ML. 

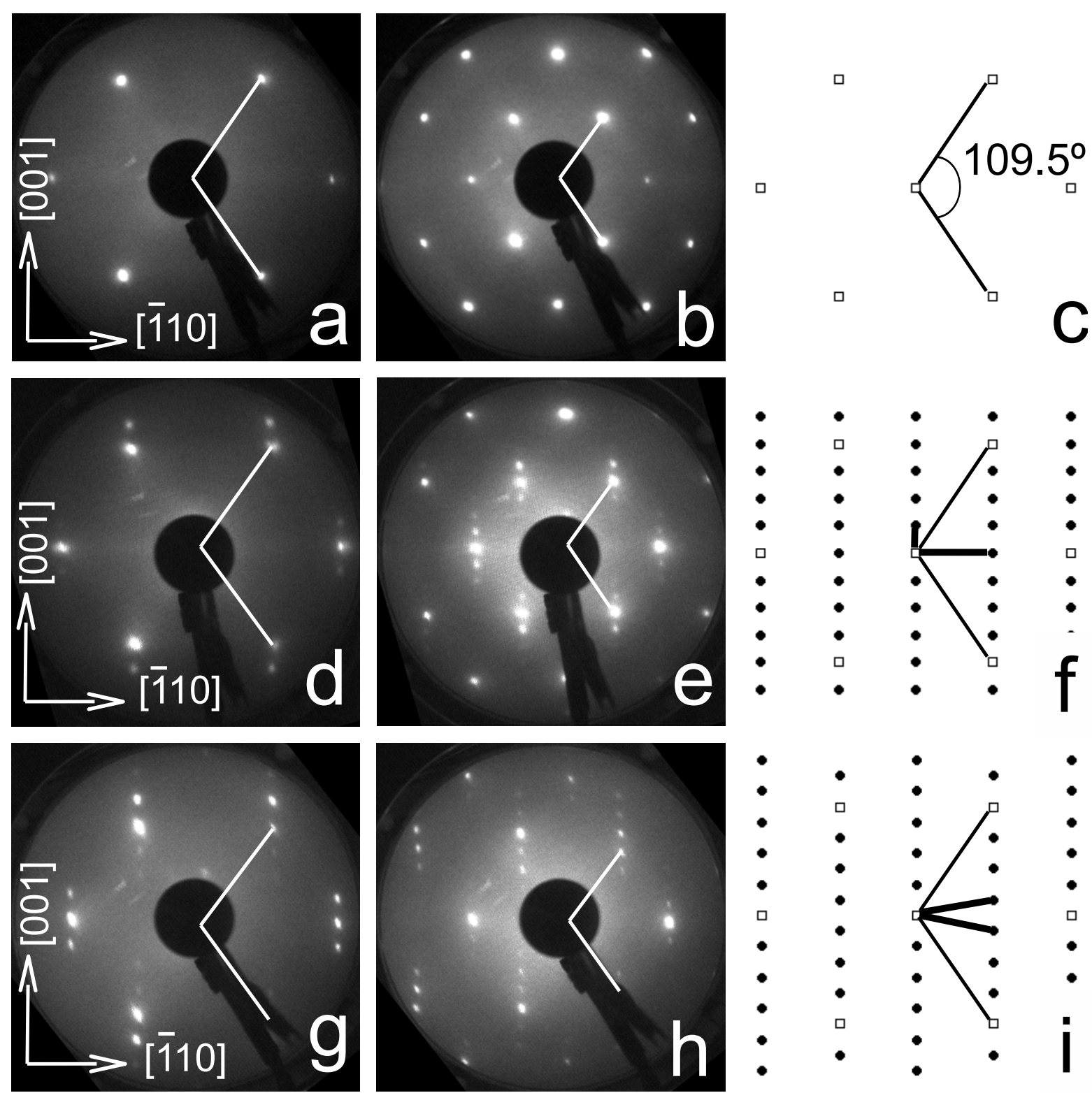

Fig. 3. LEED patterns of $\mathrm{Ni}$ films grown on $\mathrm{Mo}(110)$ at room temperature. (a) $0.2 \mathrm{ML}$ taken at $60 \mathrm{eV}$, (b) same film taken at $146 \mathrm{eV}$, and (c) a schematic model of the pattern showing the reciprocal net vectors of the substrate, which are separated by an angle of $109.5^{\circ}$. (d) $0.4 \mathrm{ML}$ taken at $60 \mathrm{eV}$, (e) same film taken at $133 \mathrm{eV}$, and (f) a schematic model showing the reciprocal net vectors of the substrate and the $(8 \times 1)$ superlattice. $(\mathrm{g}) 0.8 \mathrm{ML}$ taken at $63 \mathrm{eV}$, (h) same film taken at $116 \mathrm{eV}$, and (i) a schematic model showing the reciprocal net vectors of the substrate and the $(7 \times 1)$ superlattice. 

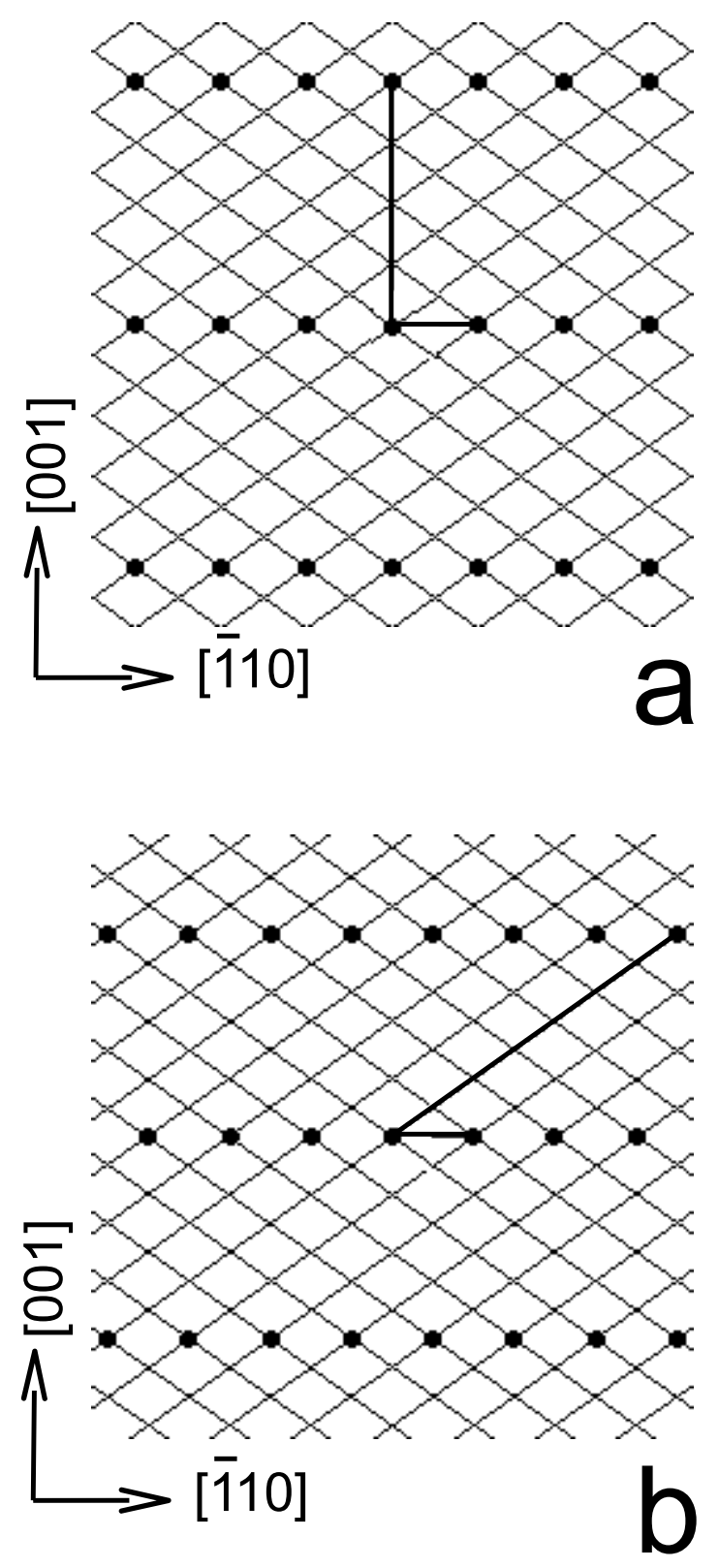

Fig. 4. Schematic models of the real space representations of the (a) $(8 \times 1)$ and $(b)$ $(7 \times 1) \mathrm{Ni} / \mathrm{Mo}(110)$ epitaxial superlattices. The $\mathrm{Mo}(110)$ surface net is indicated by a grid, while the surface net vectors of the superlattices are indicated by heavy lines. 

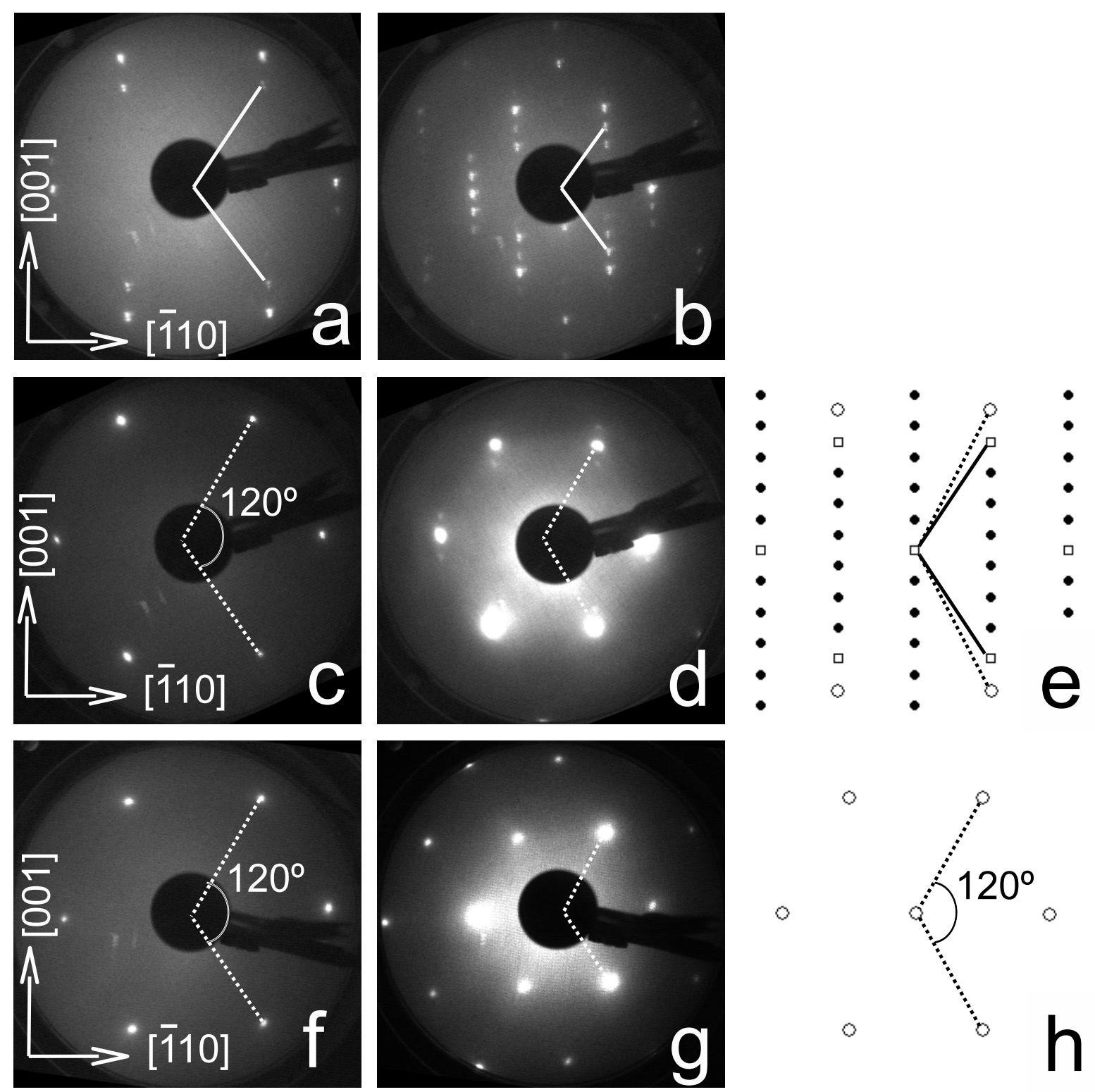

Fig. 5. LEED patterns of multilayer Ni films grown on $\mathrm{Mo}(110)$ at room temperature. (a) $1.5 \mathrm{ML}$ taken at $54 \mathrm{eV}$, (b) same film taken at $137 \mathrm{eV}$, the reciprocal net vectors for $\mathrm{Mo}(110)$ are indicated. (c) $6.2 \mathrm{ML}$ taken at $71 \mathrm{eV}$, (d) same film taken at $117 \mathrm{eV}$, and (e) a schematic model of the pattern with the reciprocal net vectors for $\mathrm{Mo}(110)$ (solid lines) and $\mathrm{Ni}(111)$ (dashed lines) indicated. (f) $9.2 \mathrm{ML}$ taken with $73 \mathrm{eV},(\mathrm{g})$ same film taken at $165 \mathrm{eV}$, and (h) a schematic model of the pattern with the reciprocal net vectors of $\mathrm{Ni}(111)$ indicated, which are separated by an angle of $120^{\circ}$. 

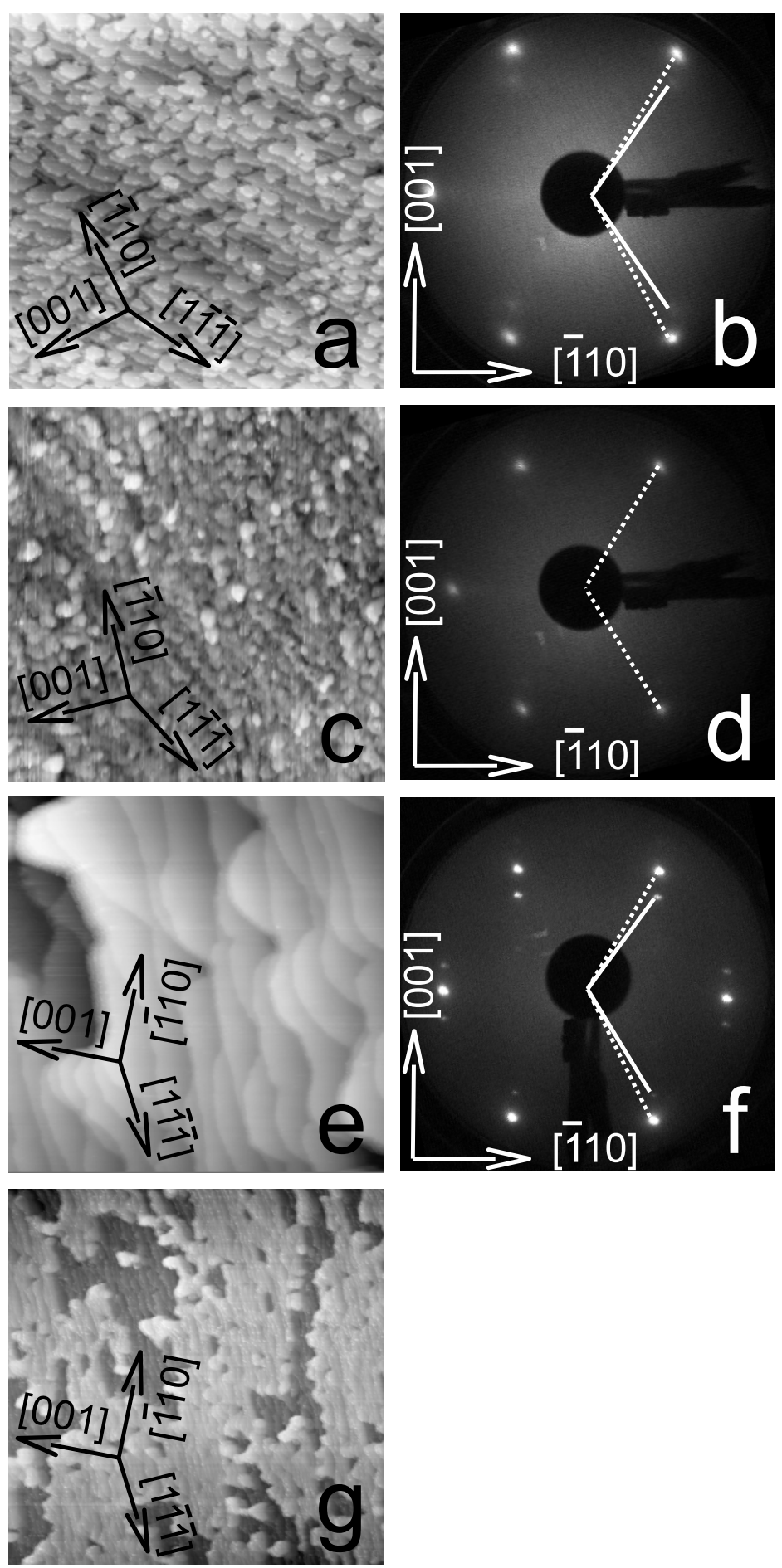

Fig. 6. (a) $200 \mathrm{~nm} \times 200 \mathrm{~nm}$ STM image of a $4.6 \mathrm{ML}$ Ni film deposited at $300 \mathrm{~K}$ on $\mathrm{Mo}(110)$. (b) LEED pattern of the same film taken at $51 \mathrm{eV}$ with the reciprocal net vectors of $\mathrm{Mo}(110)$ (solid lines) and $\mathrm{Ni}(111)$ (dashed lines) indicated. (c) $200 \mathrm{~nm} \times 200 \mathrm{~nm}$ STM image of a $4.6 \mathrm{ML} \mathrm{Ni}$ film deposited at $300 \mathrm{~K}$ and post-annealed for $1 \mathrm{~h}$ at $575 \mathrm{~K}$. (d) LEED pattern of the same film taken at $69 \mathrm{eV}$ with $\mathrm{Ni}(111)$ reciprocal net vectors indicated. (e) $200 \mathrm{~nm} \times 200 \mathrm{~nm}$ STM image of a 4.6 ML Ni film deposited at $300 \mathrm{~K}$ and post-annealed for $1 \mathrm{~h}$ at $750 \mathrm{~K}$. (f) LEED pattern of the same film taken at $65 \mathrm{eV}$. (g) $1 \mu \mathrm{m} \times 1 \mu \mathrm{m}$ STM image of same film showing agglomeration of the Ni. 

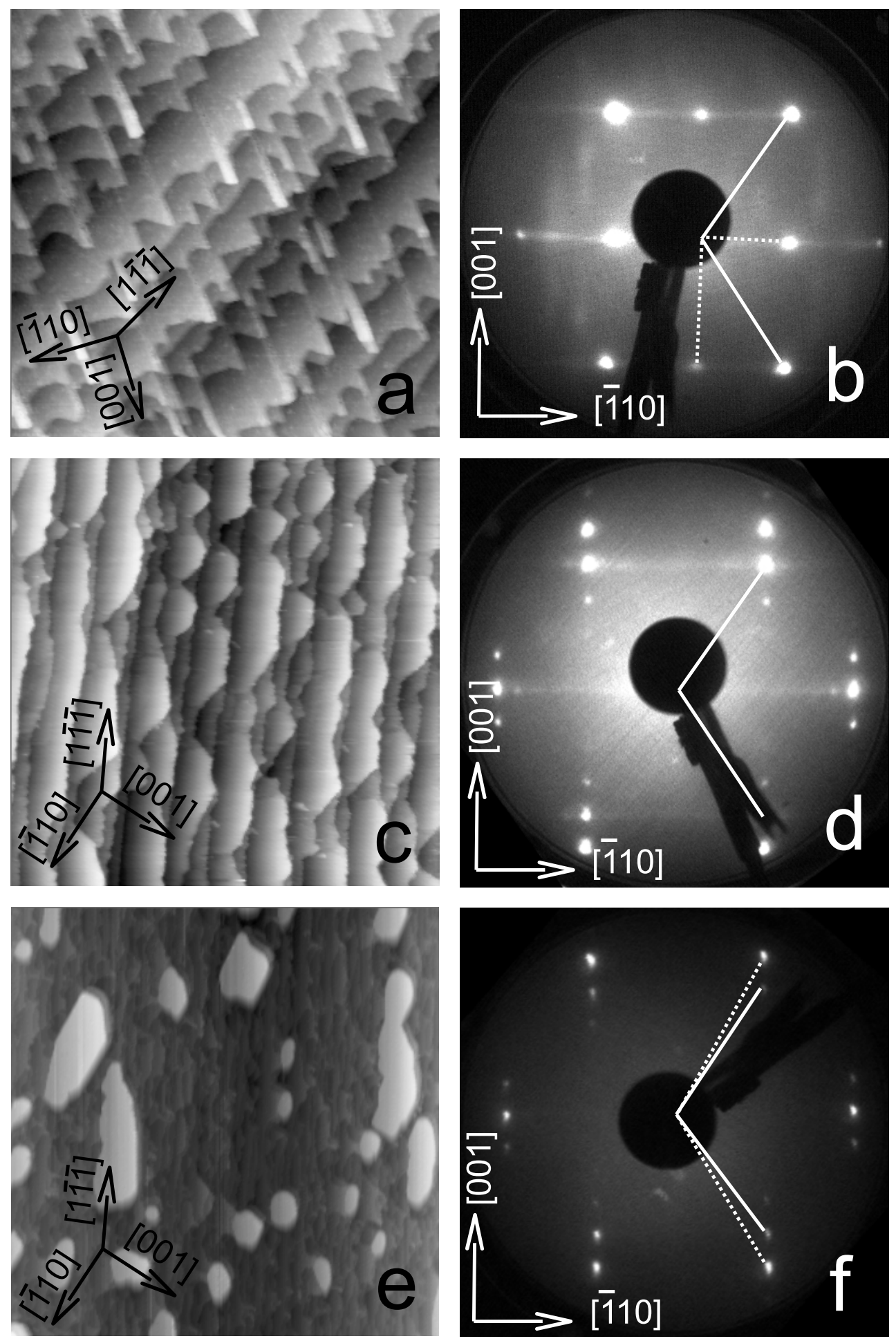

Fig. 7. (a) $500 \mathrm{~nm} \times 500 \mathrm{~nm}$ STM image of a $0.15 \mathrm{ML}$ Ni film deposited on $\mathrm{Mo}(110)$ at $300 \mathrm{~K}$ and post-annealed for $1 \mathrm{~h}$ at $855 \mathrm{~K}$. (b) c (2×2) LEED pattern of the same surface taken at $55 \mathrm{eV}$, with the reciprocal net vectors of the substrate and superlattice indicated. (b) $500 \mathrm{~nm} \times 500 \mathrm{~nm}$ STM image of a $0.8 \mathrm{ML}$ film annealed at $855 \mathrm{~K}$. (d) Corresponding LEED pattern of the surface taken at $57 \mathrm{eV}$. (e) $1 \mu \mathrm{m} \times 1 \mu \mathrm{m}$ image of a $2.3 \mathrm{ML}$ film annealed at $825 \mathrm{~K}$. (f) LEED pattern of the same surface taken at $55 \mathrm{eV}$ with the reqiprocal net vectors of $\mathrm{Mo}(110)$ (solid lines) and $\mathrm{Ni}(111)$ (dashed lines) shown. 

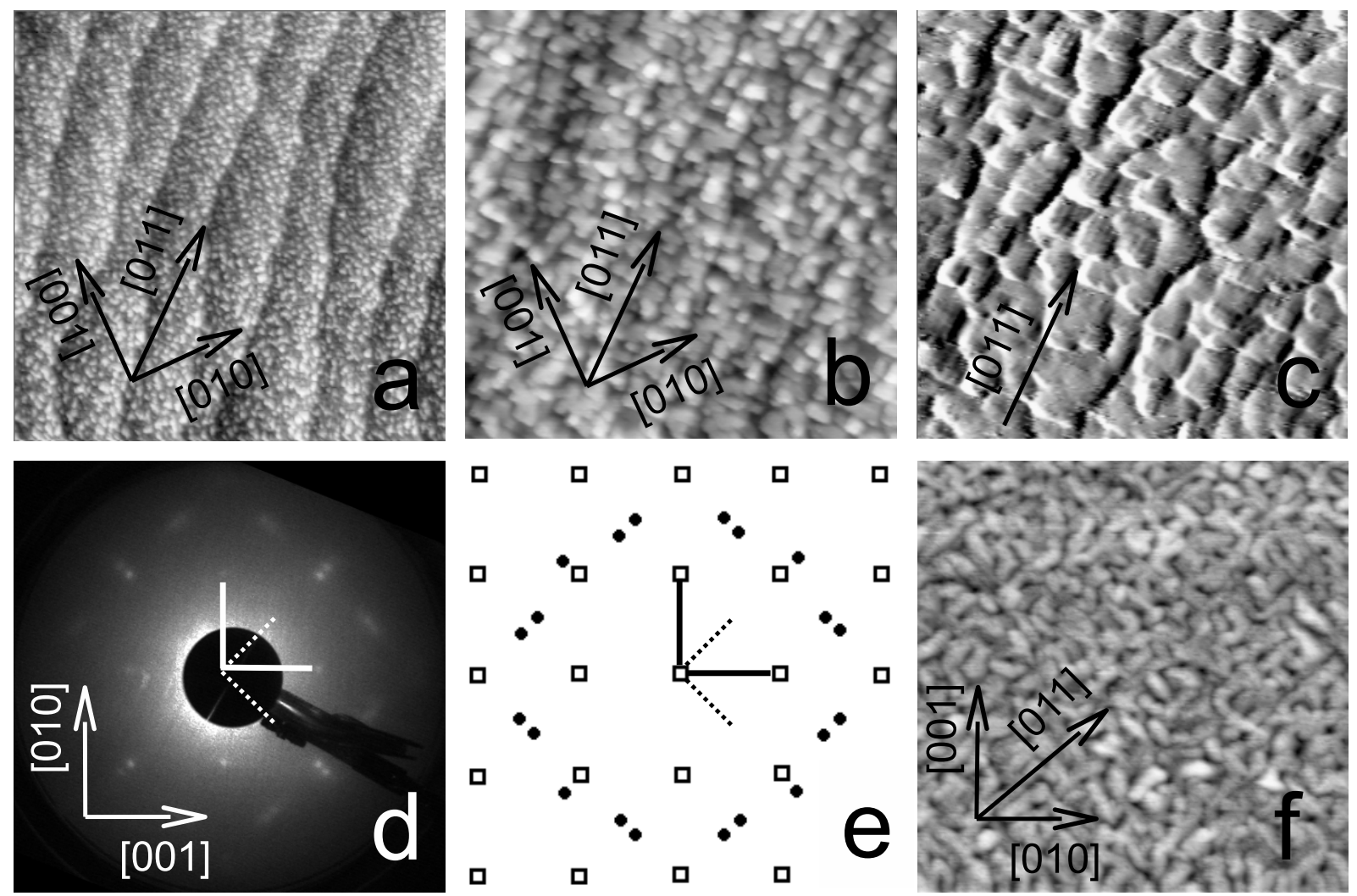

Fig. 8. Ni films deposited on $\mathrm{W}(100)$ at $300 \mathrm{~K}$. (a) $100 \mathrm{~nm} \times 100 \mathrm{~nm}$ STM image of a $0.5 \mathrm{ML}$ film, (b) $100 \mathrm{~nm} \times 100 \mathrm{~nm}$ image of a $1.8 \mathrm{ML}$ film, (c) $50 \mathrm{~nm} \times 50 \mathrm{~nm}$ differentiated image of the same film with ripples apparent on the surface of some islands. (d) LEED pattern of a $2.7 \mathrm{ML}$ film with the reciprocal net vectors of the substrate (solid lines) and film (dashed lines) indicated. (e) Schematic illustration of the LEED pattern, open squares denote integral order spots, while solid circles represent the additional reflections due to the Ni film. (f) $100 \mathrm{~nm} \times 100 \mathrm{~nm}$ STM image of a $6.8 \mathrm{ML}$ film. 